\title{
Monogenic Polynomials of Four Variables with Binomial Expansion
}

\author{
Carla Cruz ${ }^{a}$ \\ M.I. Falcão ${ }^{b}$ \\ H.R. Malonek ${ }^{a}$ \\ ${ }^{a}$ Departamento de Matemática and CIDMA, Universidade de Aveiro, Portugal \\ ${ }^{b}$ Departamento de Matemática e Aplicações and CMAT, Universidade do Minho, Portugal
}

\begin{abstract}
In the recent past one of the main concern of research in the field of Hypercomplex Function Theory in Clifford Algebras was the development of a variety of new tools for a deeper understanding about its true elementary roots in the Function Theory of one Complex Variable. Therefore the study of the space of monogenic (Clifford holomorphic) functions by its stratification via homogeneous monogenic polynomials is a useful tool. In this paper we consider the structure of those polynomials of four real variables with binomial expansion. This allows a complete characterization of sequences of 4D generalized monogenic Appell polynomials by three different types of polynomials. A particularly important case is that of monogenic polynomials which are simply isomorphic to the integer powers of one complex variable and therefore also called pseudo-complex powers.
\end{abstract}

\section{Information}

Keywords:

Monogenic polynomials, Appell sequences, pseudo-complex powers.

Original publication:

Lecture Notes in Computer Science 8579, (2014), 204-220

DOI: $10.1007 / 978-3-319-09144-0 \_15$

http://link.springer.com

\section{Introduction}

\subsection{Historical Background}

Hypercomplex Function Theory (HFT) grew up from the attempt to develop function theoretic methods for the study of functions of several real variables. The first systematic steps by using the algebra of quaternions have been done by R. Fueter $[19,20]$. He was mainly motivated by number theoretic problems (cf. [26]). The application of Clifford Algebras can already be find in his late work [21], but a first systematical treatment was only realized by $\mathrm{R}$. Delanghe and his disciples in the 70ties and 80ties of the last century and presented in book form in [5]. Since then its title Clifford Analysis is often used as synonym for the theory that grew up from Fueters quaternionic analysis as part of HFT.

Without any claim to historical rigor or completeness, it might be possible to characterize the development in the 80ies and 90ies of the last century by the following observation. The use of representation theoretical and analytical methods succeeded to cover a wide range of applications of Clifford Analysis in different areas of Mathematics and Physics, particularly in Quantum Physics (see [16]). Another focus was on the development of a hypercomplex singular integral operator theory and a discrete quaternionic calculus for the solution of concrete problems in partial differential equations (see [23]).

The first manual written originally in German by three well known specialist in the field, was published 2006 under the title Funktionentheorie in der Ebene und im Raum. Its translation to English appeared in 2008 as Holomorphic functions in the plane and n-dimensional space. As the title indicates, this book [24] treats holomorphic functions in the plane and $n$-dimensional space in parallel and allows an interesting encounter 
with the historical motivation and dimension of HFT. It includes new results obtained in the 90ties and the first decade of our century about new insights into the roots of HFT in complex analysis which suggested such a treatment (cf. [27, 28]).

Concerning the construction and the use of Appell sequences in Clifford Analysis, we mention the works $[17,18]$ and [8] whose approach clarified some longstanding problems about the existence of monogenic polynomials as equivalent for monogenic power-like functions ([32]). The approach relied essentially on the use of the hypercomplex derivative (see Subsect. 1.2). The concept of generalized Appell sequences was later studied by representation theoretical methods (cf. [15, 25]) and further extended in [4]. The references in the latter article describe well the study about generalized Appell sequences until five years ago.

Later on, we called attention in several papers $([10,11,12,32])$ to the relationship of Appell polynomials with an old concept of totally regular variables, first mentioned by R. Delanghe in [14]. Moreover, we discussed also the possibility of constructing pseudo-complex bases of pseudo-complex powers (see Sect. 2) for monogenic polynomials as the most simple, in our opinion, building blocks of monogenic functions that conserve the structure of the complex variable $z$ and its integer powers $z^{n}$. To complete the 3D-case mainly studied so far, the present article calls attention to some new facts in the 4D-case. Numerical advantages of our approach to the 3D-case are discussed in our second contribution to this conference: On Numerical Aspects of PseudoComplex Powers in $\mathbb{R}^{3}$. Finally, the recent paper [13] shows that pseudo-complex powers are also relevant for combinatorial problems and open new fields for applications of HFT.

\subsection{Basic Notations}

In the following we use the same notations as in [32]. Therefore let $\left\{e_{1}, e_{2}, \ldots, e_{n}\right\}$ be an orthonormal basis of the Euclidean vector space $\mathbb{R}^{n}$ with the non-commutative product according to the multiplication rules

$$
e_{k} e_{l}+e_{l} e_{k}=-2 \delta_{k l}, k, l=1, \cdots, n,
$$

where $\delta_{k l}$ is the Kronecker symbol. The set $\left\{e_{A}: A \subseteq\{1, \cdots, n\}\right\}$ with

$$
e_{A}=e_{h_{1}} e_{h_{2}} \cdots e_{h_{r}}, 1 \leq h_{1}<\cdots<h_{r} \leq n, e_{\emptyset}=e_{0}=1,
$$

forms a basis of the $2^{n}$-dimensional Clifford algebra $C \ell_{0, n}$ over $\mathbb{R}$. Let $\mathbb{R}^{n+1}$ be embedded in $C \ell_{0, n}$ by the identification of the vector $\left(x_{0}, \vec{x}\right)=\left(x_{0}, x_{1}, \cdots, x_{n}\right) \in \mathbb{R}^{n+1}$ with the so-called paravector

$$
x=x_{0}+\underline{x} \in \mathcal{A}:=\operatorname{span}_{\mathbb{R}}\left\{1, e_{1}, \ldots, e_{n}\right\} \subset C \ell_{0, n} .
$$

Here $x_{0}=\operatorname{Sc}(x)$ and $\underline{x}=\operatorname{Vec}(x)=e_{1} x_{1}+\cdots+e_{n} x_{n}$ usually are called scalar resp. vector part of the paravector $x \in \mathcal{A}$. The conjugate of $x$ is given by $\bar{x}=x_{0}-\underline{x}$. Its norm is, like in the complex case, the square root of the product of $x$ and $\bar{x}$, i.e. $|x|=(x \bar{x})^{\frac{1}{2}}=\left(x_{0}^{2}+x_{1}^{\frac{2}{2}}+\cdots+x_{n}^{2}\right)^{\frac{1}{2}}$.

To call attention to its relation with the complex Wirtinger derivatives, we use for the generalized CauchyRiemann operator in $\mathbb{R}^{n+1}, n \geq 1$, the notation

$$
\bar{\partial}:=\frac{1}{2}\left(\partial_{0}+\partial_{\underline{x}}\right), \quad \partial_{0}:=\frac{\partial}{\partial x_{0}}, \quad \partial_{\underline{x}}:=e_{1} \frac{\partial}{\partial x_{1}}+\cdots+e_{n} \frac{\partial}{\partial x_{n}} .
$$

All $\mathscr{C}^{1}$-functions $f$ satisfying the equation $\bar{\partial} f=0$ (resp. $f \bar{\partial}=0$ ) are called left monogenic (resp. right monogenic). Also the expressions left Clifford holomorphic or right Clifford holomorphic are in use (see [24]). We suppose that $f$ is hypercomplex differentiable in $\Omega$ in the sense of [22], [27], i.e. has a uniquely defined generalized areolar derivative $f^{\prime}$ in the sense of Pompeiu in each point of $\Omega$ (see also [28]). Then $f$ is real differentiable and $f^{\prime}$ can be expressed by the real partial derivatives as $f^{\prime}=\partial f$ where analogously to the generalized Cauchy-Riemann operator we use $\partial:=\frac{1}{2}\left(\partial_{0}-\partial_{\underline{x}}\right)$ for the conjugate Cauchy-Riemann operator. Using the fact that a hypercomplex differentiable function belongs to the kernel of $\bar{\partial}$, it follows that in fact $f^{\prime}=\partial_{0} f=-\partial_{\underline{x}} f$ which is the generalization of the complex case $n=1$ with $e_{1} \cong i$.

In general, $C \ell_{0, n}$-valued functions defined in some open subset $\Omega \subset \mathbb{R}^{n+1}$ are of the form $f(z)=$ $\sum_{A} f_{A}(z) e_{A}$, with real valued $f_{A}(z)$. In the special case of $\mathcal{A}$-valued monogenic functions of the paravector $x \in \mathcal{A}$ that we are going to study we have

$$
f\left(x_{0}, \underline{x}\right)=\sum_{j=0}^{n} f_{j}\left(x_{0}, \underline{x}\right) e_{j} .
$$


It is worth noting that in this case left monogenic $(\bar{\partial} f=0)$ functions are also right monogenic functions $(f \bar{\partial}=0)$. This fact follows easily by direct inspection of the two corresponding real systems of first order partial differential equations. In [33] such generalized Cauchy-Riemann system is also called Riesz system and its solutions have been studied in context with the representation of the group of rotations in $\mathbb{R}^{4}$.

We mention also that the study of monogenic functions has led to a refinement of several results in Harmonic Analysis. The reason relies on the fact that both Cauchy-Riemann operators factorize the Laplace operator $\Delta_{(4)}$ in the form $\Delta_{(4)}=4 \bar{\partial} \partial=4 \partial \bar{\partial}$, which means that all real components of a monogenic functions are harmonic functions. Again we can recognize the close relationship between Hypercomplex Function Theory and the Function Theory of one Complex Variable with respect to the common properties of the main function classes studied in both theories.

\section{Generalized Appell Polynomials and Pseudo-complex Power Func- tions}

\subsection{A Sequence of Standard Appell Polynomials (SAP)}

Bernoulli, Euler, Hermite and other polynomials over the field of complex numbers are well known examples of Appell polynomials. The introduction of the concept of Appell sequences into the framework of Clifford Analysis some years ago was one way towards a deeper understanding of the structure of monogenic polynomials (see Subsect. 1.1). Because of the usually considered stratification of the monogenic function space via homogeneous monogenic polynomials, special attention was paid to the structure of those homogeneous monogenic polynomials. But even after so long time, the difficulties caused by the non-commutative Clifford algebra let some questions open.

That is also why the main subject of our studies is the complete characterization of generalized sequences of Appell polynomials in four real variables. Continuing our recent work about the corresponding case of three variables, we detected in the case of four variables a new type of generalized Appell polynomials, different from those in the case of three variables. As we will show in the last section, the new type has a more complicated structure than the so-called Standard Appell Polynomials or the pseudo-complex power functions which are simply isomorphic to the integer powers of one complex variable and therefore exist in any dimension.

In this work we are particularly concerned with the proof of a conjecture in [10] about the coefficients of the third type of Appell polynomials in four variables. Indeed, it might be that an increasing number of variables implies an increasing number of new types of generalized Appell polynomials unknown so far. But to prove this, probably other methods have to be developed.

We refer now to some details about a special type of Appell polynomials which is known for any number of variables. First we recall the classical definition by Appell in [3] adapted to the hypercomplex case and the hypercomplex derivative.

Definition 1 A sequence of monogenic polynomials $\left(P_{k}\right)_{k \geq 0}$ of exact degree $k$ is called a generalized Appell sequence with respect to $\partial$ if

1. $P_{0}(x) \equiv 1$,

2. $\partial P_{k}=k P_{k-1}, k=1,2, \ldots$

We note that the first is the usually applied normalization condition and can be changed to any constant different from zero. In the following we consider only generalized Appell sequences with polynomials of zero degree of the form $P_{0}(x) \equiv 1$. The paper [2] studies 3D generalized Appell sequences with a general $\mathcal{A}-$ valued constant as zero degree polynomial $P_{0}(x)$.

An equivalent definition through the property of a binomial expansion is provided by:

Proposition 1 (Theorem 1,[6]) A monogenic polynomial sequence $\left(P_{k}\right)_{k \geq 0}$ is an Appell set if and only if it satisfies the binomial expansion

$$
P_{k}(x)=P_{k}\left(x_{0}+\underline{x}\right)=\sum_{s=0}^{k}\left(\begin{array}{l}
k \\
s
\end{array}\right) P_{k-s}\left(x_{0}\right) P_{s}(\underline{x}), x \in \mathcal{A} .
$$


The so-called standard homogeneous Appell polynomials (SAP) $([6,7,18])$ are well known, but we introduced its designation SAP only for simplification of writing. Independently from the number of variables they can be written as

$$
\mathcal{P}_{k}\left(x_{0}, \underline{x}\right)=\sum_{s=0}^{k}\left(\begin{array}{l}
k \\
s
\end{array}\right) c_{s}(n) x_{0}^{k-s} \underline{x}^{s},
$$

where

$$
c_{s}(n)= \begin{cases}\frac{s ! !(n-2) ! !}{(n+s-1) ! !}, & \text { if } s \text { is odd, } \\ c_{s-1}(n), & \text { if } s \text { is even, }\end{cases}
$$

and, as usual, we define $(-1) ! !=0 ! !=1$.

We notice also a remarkable fact that for the first noncommutative case, i.e. when $n=2$ and $\underline{x}=$ $x_{1} e_{1}+x_{2} e_{2}$, the coefficients $c_{s}(2)$ are the generalized central binomial coefficients with weight $\frac{1}{2^{s}}$, i.e.,

$$
c_{s}(2)=\frac{1}{2^{s}}\left(\begin{array}{c}
s \\
\left\lfloor\frac{s}{2}\right\rfloor
\end{array}\right), \quad s=0,1, \ldots, k,
$$

where $\lfloor\cdot\rfloor$ is the floor function. Moreover, as it will be shown in Theorem 2, the coefficients $c_{s}(2)$ appear again in particular cases of Appell polynomials with four variables.

The formula reveals some special values in the case $n=3$ with $\underline{x}=x_{1} e_{1}+x_{2} e_{2}+x_{3} e_{3}$. The $c_{s}(3)$ are nothing else than the reciprocal values of all odd integers. That is, we have $c_{0}(3)=1$ and

$$
c_{s}(3)=\frac{1}{s+2}=c_{s+1}(3) \text { for } s=1,3,5, \ldots
$$

We studied in the past some applications of SAP's in the construction of quasi-conformal mappings in the space in [29] and the approximation of quasi-conformal mappings in [31]. Exactly the same Appell polynomials (2) appeared later on in connection with other applications studied by other authors, for example in [4, 15, 25].

Notice that SAP's form a subset of Special Appell Polynomials which have been introduced in [1] as monogenic polynomials $P$ with coefficients $a_{i j} \in \mathcal{A}_{n}$ and representation in the form of

$$
P(x)=\sum_{i, j}^{\prime} \bar{x}^{i} x^{j} a_{i, j},
$$

where the primed sigma stands for a finite sum. Of course, writing $x_{0}=\frac{1}{2}(x+\bar{x})$ and $\underline{x}=\frac{1}{2}(x-\bar{x})$ we see that a rearrangement of (2) leads to the form (4) (see also [30] about different representations of SAP's).

\subsection{Pseudo-complex Powers (PCP)}

The second type of generalized Appell polynomials can be introduced in the following way.

Definition 2 Consider $\vec{i}=\left(i_{1}, \ldots, i_{n}\right) \in S^{n-1}$, its scalar product with $\vec{x} \in \mathbb{R}^{n}$ in form of $x_{\hat{\imath}}:=\vec{i} \vec{x}=$ $\left(i_{1} x_{1}+\cdots+i_{n} x_{n}\right)$, and the associated hypercomplex (pure) paravector $\hat{\imath}=i_{1} e_{1}+\cdots+i_{n} e_{n}$. The linear function $Z_{\hat{\imath}}: \mathcal{A} \rightarrow \mathbb{C}_{\hat{\imath}}$, with $\mathbb{C}_{\hat{\imath}}:=\operatorname{span}_{\mathbb{R}}\{1, \hat{\imath}\} \subset C \ell_{0, n}$ and given by

$$
Z_{\hat{\imath}}=Z_{\hat{\imath}}\left(x_{0}, \vec{x}\right):=x_{0}+\hat{\imath} x_{\hat{\imath}}=x_{0}+\left(i_{1} e_{1}+\cdots+i_{n} e_{n}\right)\left(i_{1} x_{1}+\cdots+i_{n} x_{n}\right)
$$

defines a variable which we designate by pseudo-complex variable(PCV).

Note that the exact designation of this variable should include the dimension $n$ of the space. However, for simplicity we avoid here to do so.

In the case of $\vec{i}_{k}=(0, \ldots, 1, \ldots, 0),(1$ in the $k$-th coordinate and 0 elsewhere), the corresponding PCV's have as imaginary unit the basic element $\hat{\imath}_{k}=e_{k}$ and are the $n$ copies of the ordinary complex variable $z=x+i y$, i.e.

$$
Z_{\hat{\imath}_{k}}=x_{0}+x_{k} e_{k}=e_{k} z_{k} .
$$

Here we mention on the right hand side of (6) the expression of $Z_{\hat{\imath}_{k}}$ in terms of the Fueter variable $z_{k}:=$ $x_{k}-x_{0} e_{k} ; k=1, \ldots n$, which plays an important role in the representation of monogenic functions by generalized power series (cf. [24]). 
Representations of $Z_{\hat{\imath}}$ other than (5) are, for example:

$$
Z_{\hat{\imath}}=\left(x_{\hat{\imath}}-x_{0} \hat{\imath}\right) \hat{\imath}=\left(z_{1} i_{1}+\cdots+z_{n} i_{n}\right) \hat{\imath} .
$$

While in the case of (5) the character of $Z_{\hat{\imath}}$ as a special paravector is reflected, the relation (7) stresses its connection with the Fueter variables $z_{k}$.

Proposition 2 Because of the isomorphism property, an integer power of order $k$ of a PCV $Z_{\hat{\imath}}$ is monogenic and can be written, at least in two ways as

$$
\begin{aligned}
Z_{\hat{\imath}}^{k} & =\left(Z_{\hat{\imath}}\right)^{k}=\left(x_{0}+\hat{\imath} x_{\hat{\imath}}\right)^{k}, \\
& =\left(z_{1} i_{1}+\cdots+z_{n} i_{n}\right)^{k} \hat{\imath}^{k} .
\end{aligned}
$$

In the following we will refer to $Z_{\hat{\imath}}^{k}$ as a pseudo-complex power (PCP) of order $k$.

Furthermore, we have an easy consequence of the isomorphism property:

Proposition 3 ([8]) For each $\hat{\imath}=i_{1} e_{1}+\cdots+i_{n} e_{n}$, such that $\hat{\imath}^{2}=-1$, the sequence $\left(Z_{\hat{\imath}}^{k}\right)_{k \geq 0}$ is an Appell sequence.

Besides emphasizing the isomorphism between the property of the ordinary complex powers $z^{k}$ and the PCP's $Z_{\hat{\imath}}^{k}$, both sequences share the condition that their restriction to the real line (i.e. $y=0$ resp. $\underline{x}=0$ ) is the ordinary real Appell sequence $\left(x_{0}^{k}\right)_{k>0}$. This observation motivated the following question:

Are the SAP and the PCP the only types of monogenic Appell polynomials such that their restriction to $\underline{x}=0$ is $x_{0}^{k} ?$

\section{Homogeneous Monogenic Polynomials of Four Variables with Bi- nomial Expansion}

In order to study this problem we use the binomial expansion (1), but now with a general homogeneous linear vector part

$$
\underline{X}=X_{1}\left(x_{1}, \ldots, x_{n}\right) e_{1}+\cdots+X_{n}\left(x_{1}, \ldots, x_{n}\right) e_{n},
$$

which for $\underline{x}=0$ vanishes and warranted, together with the binomial expansion, that $P_{k}\left(x_{0}, \underline{0}\right)=x_{0}^{k}$. In fact, we consider

$$
P_{k}\left(x_{0}, \underline{x}\right)=\sum_{s=0}^{k}\left(\begin{array}{l}
k \\
s
\end{array}\right) d_{s}(n) x_{0}^{k-s} \underline{X}^{s},
$$

where $d_{s}(n)$ are suitably chosen real coefficients.

It is easy to recognize that the polynomials (10) have values in $\mathcal{A}$ since $\underline{X}^{s}$ is real valued or pure vector valued, depending on whether $s$ is even or odd, respectively. As already mentioned, left monogenic functions of the form (10) are also right monogenic and, consequently, it is enough to solve the real system of first order partial differential equations corresponding to $\bar{\partial} P_{k}=0$. Moreover, with the additional assumption $d_{0}(n) \equiv 1$ we have guaranteed the normalization condition $P_{k}\left(x_{0}, \underline{0}\right)=x_{0}^{k}$.

In the case $n=2$, the complete characterization of polynomials with three variables, of the form (10) is possible as we will briefly recall. By direct inspection of $\bar{\partial} P_{1}=0$ with $\partial P_{1}=P_{0}=1$, the first degree polynomial $P_{1}$ is monogenic if and only if it has the form

$$
P_{1}\left(x_{0}, x_{1} e_{1}+x_{2} e_{2}\right)=x_{0}+d_{1}\left(\left(a_{1} x_{1}+\lambda x_{2}\right) e_{1}+\left(\lambda x_{1}+a_{2} x_{2}\right) e_{2}\right),
$$

with $a_{1}, a_{2}, \lambda \in \mathbb{R}$ and

$$
d_{1}=\frac{1}{a_{1}+a_{2}}, \quad \text { for } \quad a_{1}+a_{2} \neq 0 .
$$

Because of (11) only one of the coefficients $a_{1}$ or $a_{2}$ can be equal to zero and these both exceptional cases together with the Appell condition $\partial P_{2}=2 P_{1}$ lead directly to the trivial cases, that

$$
P_{1}\left(x_{0}, x_{1} e_{1}+x_{2} e_{2}\right)=x_{0}+x_{1} e_{1} \quad \text { or } \quad P_{1}\left(x_{0}, x_{1} e_{1}+x_{2} e_{2}\right)=x_{0}+x_{2} e_{2} .
$$


Moreover, if $a_{2}=0$, then

$$
P_{k}\left(x_{0}, \underline{x}\right)=P_{k}\left(x_{0}, x_{1} e_{1}\right)=\sum_{s=0}^{k}\left(\begin{array}{l}
k \\
s
\end{array}\right) x_{0}^{k-s}\left(x_{1} e_{1}\right)^{s}=\left(x_{0}+x_{1} e_{1}\right)^{k} .
$$

Analogously, if $a_{1}=0$, then

$$
P_{k}\left(x_{0}, \underline{x}\right)=P_{k}\left(x_{0}, x_{2} e_{2}\right)=\sum_{s=0}^{k}\left(\begin{array}{l}
k \\
s
\end{array}\right) x_{0}^{k-s}\left(x_{2} e_{2}\right)^{s}=\left(x_{0}+x_{2} e_{2}\right)^{k} .
$$

Resuming: If $a_{1}+a_{2} \neq 0$ and $a_{1} a_{2}=0$, then the corresponding Appell polynomials are trivial copies of the complex case with $i \cong e_{1}$ or $i \cong e_{2}$.

The non-trivial cases corresponding to $a_{1}+a_{2} \neq 0$ and $a_{1} a_{2} \neq 0$, are described in the following theorem:

Theorem 1 (Theorem 2.1, [32]) There exist only two different types of non-trivial Appell polynomials of the general form

$$
P_{k}\left(x_{0}, \underline{x}\right)=\sum_{s=0}^{k}\left(\begin{array}{l}
k \\
s
\end{array}\right) d_{s} x_{0}^{k-s}\left(X_{1} e_{1}+X_{2} e_{2}\right)^{s}
$$

namely

1. The SAP (2)

$$
P_{k}\left(x_{0}, \underline{x}\right)=\mathcal{P}_{k}^{n}\left(x_{0}, \underline{x}\right)=\sum_{s=0}^{k}\left(\begin{array}{l}
k \\
s
\end{array}\right) c_{s}(2) x_{0}^{k-s} \underline{x}^{s} .
$$

2. The PCP (8)

$$
P_{k}\left(x_{0}, \underline{x}\right)=Z_{\hat{\imath}}^{k}=\sum_{s=0}^{k}\left(\begin{array}{l}
k \\
s
\end{array}\right) x_{0}^{k-s}\left(i_{1} x_{1}+i_{2} x_{2}\right)^{s} \hat{\imath}^{s},
$$

where $\hat{\imath}^{2}=\left(i_{1} e_{1}+i_{2} e_{2}\right)^{2}=-1$.

Theorem 1 shows the exclusive role played by the SAP and the PCP's in the context of Appell polynomials in $\mathbb{R}^{3}$ restricted to the condition $d_{0}(2)=1$. In [10] the authors started to extend this result by considering the corresponding $n=3$ form of the polynomials (10), i. e.,

$$
P_{k}\left(x_{0}, \underline{x}\right)=\sum_{s=0}^{k}\left(\begin{array}{l}
k \\
s
\end{array}\right) d_{s}(3) x_{0}^{k-s}\left(X_{1} e_{1}+X_{2} e_{2}+X_{3} e_{3}\right)^{s} .
$$

For the sake of better understanding, we reproduce here the main result of [10]. From now on we use briefly $d_{s}:=d_{s}(3), s \geq 0$. In this case the first degree polynomial $P_{1}\left(x_{0}, \underline{x}\right)=x_{0}+d_{1} \underline{X}$ is monogenic if the real linear functions $X_{j}$ are of the form

$$
X_{1}=a_{1} x_{1}+\lambda_{1} x_{2}+\lambda_{2} x_{3}, X_{2}=\lambda_{1} x_{1}+a_{2} x_{2}+\lambda_{3} x_{3}, X_{3}=\lambda_{2} x_{1}+\lambda_{3} x_{2}+a_{3} x_{3},
$$

where $a_{1}, a_{2}, a_{3}$ are real parameters such that

$$
a_{1}+a_{2}+a_{3} \neq 0
$$

and the coefficient $d_{1}$ is given by

$$
d_{1}=\frac{1}{a_{1}+a_{2}+a_{3}} .
$$

It follows immediately that the special cases

$$
\lambda_{1}=\lambda_{2}=a_{1}=0 \quad \text { or } \quad \lambda_{1}=\lambda_{3}=a_{2}=0 \quad \text { or } \quad \lambda_{2}=\lambda_{3}=a_{3}=0
$$


correspond to 3D polynomials and therefore Theorem 1 holds, i.e. (12) are, in fact, 3D standard Appell polynomials, 3D pseudo-complex polynomials or complex powers.

We proceed now by examining under what conditions the second degree polynomial $P_{2}\left(x_{0}, \underline{x}\right)=x_{0}+$ $2 d_{1} \underline{X}+d_{2} \underline{X}^{2}$ is monogenic. This problem is equivalent, for independent $x_{1}, x_{2}$ and $x_{3}$, to solve the nonlinear system

$$
\left\{\begin{array}{l}
a_{1}-d_{2}\left(a_{1}^{2}+\lambda_{1}^{2}+\lambda_{2}^{2}\right)\left(a_{1}+a_{2}+a_{3}\right)=0 \\
a_{2}-d_{2}\left(\lambda_{1}^{2}+a_{2}^{2}+\lambda_{3}^{2}\right)\left(a_{1}+a_{2}+a_{3}\right)=0 \\
a_{3}-d_{2}\left(\lambda_{2}^{2}+\lambda_{3}^{2}+a_{3}^{2}\right)\left(a_{1}+a_{2}+a_{3}\right)=0 \\
\lambda_{1}-d_{2}\left(\lambda_{1}\left(a_{1}+a_{2}\right)+\lambda_{2} \lambda_{3}\right)\left(a_{1}+a_{2}+a_{3}\right)=0 \\
\lambda_{2}-d_{2}\left(\lambda_{2}\left(a_{1}+a_{3}\right)+\lambda_{1} \lambda_{3}\right)\left(a_{1}+a_{2}+a_{3}\right)=0 \\
\lambda_{3}-d_{2}\left(\lambda_{3}\left(a_{2}+a_{3}\right)+\lambda_{1} \lambda_{2}\right)\left(a_{1}+a_{2}+a_{3}\right)=0
\end{array}\right.
$$

Cumbersome, but straightforward calculations lead to the following essential cases:

1. $\lambda_{1}=\lambda_{2}=\lambda_{3}=0$

We can assume that $a_{1} a_{2} a_{3} \neq 0$, since the trivial case where one of the $a_{i}$ 's is zero was already considered in (13). The solution of (14) is:

$$
a_{1}=a_{2}=a_{3}=\alpha \quad(\alpha \neq 0) \quad \text { and } \quad d_{2}=\frac{1}{3 \alpha^{2}} .
$$

In this case, since $d_{1}=\frac{1}{3 \alpha}$ it follows immediately that

$$
P_{1}\left(x_{0}, \underline{x}\right)=\mathcal{P}_{1}(x)=x_{0}+\frac{1}{3} \underline{x} .
$$

Furthermore, $\underline{X}=\alpha\left(x_{1} e_{1}+x_{2} e_{2}+x_{3} e_{3}\right)=\alpha \underline{x}$, which allows to express $P_{k}$ as

$$
P_{k}\left(x_{0}, \underline{x}\right)=\sum_{s=0}^{k}\left(\begin{array}{l}
k \\
s
\end{array}\right) d_{s} \alpha^{s} x_{0}^{k-s} \underline{x}^{s} .
$$

If we now apply Theorem 2 of [7], where homogeneous monogenic polynomials expressed in terms of powers of $x_{0}$ and $\underline{x}$ were completely characterized, then we conclude that

$$
d_{s}=\frac{c_{s}(3)}{\alpha^{s}}, s=0, \ldots, k
$$

In other words, this case corresponds to the SAP (cf. (2)) in $\mathbb{R}^{4}$.

2. $\lambda_{1} \neq 0$ or $\lambda_{2} \neq 0$ or $\lambda_{3} \neq 0$

These cases correspond to assuming that either one of the parameters $\lambda_{k}, k=1,2,3$ is nonzero and the other two vanish or all the parameters are nonzero. The situation where two of the parameters are nonzero and the other one is zero is not possible (see equations 4-6 of (14)).

If one of the parameters is nonzero and the other two vanish, the system (14) reduces essentially and can be solved easily with respect to $d_{2}$. It leads to 3D polynomials as one of the cases of (13) (where the corresponding coefficient $a_{k}, k=1,2,3$ is assumed of being equal to zero) or to a solution of the form $d_{2}=2 d_{1}^{2}$ (where the corresponding coefficient $a_{k}, k=1,2,3$ is not zero, but must be equal to the sum of the other two $a_{k}$ ). Because of $d_{2}=2 d_{1}^{2}$ the binomial expansion of the Appell polynomial corresponds to a particular case of a 4D polynomial that will be treated next in ii.

In the case $\lambda_{1} \lambda_{2} \lambda_{3} \neq 0$, the system (14) leads to the quadratic equation in $d_{2}$ :

$$
\left(a_{1}+a_{2}+a_{3}\right)^{4} d_{2}^{2}-3\left(a_{1}+a_{2}+a_{3}\right)^{2} d_{2}+2=0,
$$

which admits two different solutions: 

i. $\quad d_{2}=\frac{1}{\left(a_{1}+a_{2}+a_{3}\right)^{2}}=d_{1}^{2}$
or
ii. $\quad d_{2}=\frac{2}{\left(a_{1}+a_{2}+a_{3}\right)^{2}}=2 d_{1}^{2}$

In what follows we analyze both cases separately.

i. In this case we conclude from (14) that $\lambda_{1}, \lambda_{2}, \lambda_{3}$ are roots of

$$
\lambda_{1}^{2}=a_{1} a_{2}, \quad \lambda_{2}^{2}=a_{1} a_{3}, \quad \lambda_{3}^{2}=a_{2} a_{3},
$$

chosen so that $\lambda_{1} \lambda_{2} \lambda_{3}=a_{1} a_{2} a_{3}$. We point out that, due to (15), if $a_{1}, a_{2}$ and $a_{3}$ are nonzero, then they must have the same sign. Following the technique of [11], we introduce now the real parameters

$$
i_{1}^{2}:=\frac{a_{1}}{a_{1}+a_{2}+a_{3}}, \quad i_{2}^{2}:=\frac{a_{2}}{a_{1}+a_{2}+a_{3}}, \quad i_{3}^{2}:=\frac{a_{3}}{a_{1}+a_{2}+a_{3}},
$$

which allows to write

$$
P_{1}\left(x_{0}, \underline{x}\right)=x_{0}+\left(i_{1} x_{1}+i_{2} x_{2}+i_{3} x_{3}\right)\left(i_{1} e_{1}+i_{2} e_{2}+i_{3} e_{3}\right)
$$

for a chosen triplet of those roots $i_{1}, i_{2}$ and $i_{3}$ as defined in (16). Notice that from this relation we obtain $i_{1}^{2}+i_{2}^{2}+i_{3}^{2}=1$ and it follows immediately that $\left(i_{1} e_{1}+i_{2} e_{2}+i_{3} e_{3}\right)^{2}=-1$. In other words, we obtain the 4D-version of the PCP's (8).

ii. In this situation (14) allows to conclude that $\lambda_{1}, \lambda_{2}, \lambda_{3}$ are roots of

$$
\lambda_{1}^{2}=\frac{1}{4} A_{1} A_{2}, \quad \lambda_{2}^{2}=\frac{1}{4} A_{1} A_{3}, \quad \lambda_{3}^{2}=\frac{1}{4} A_{2} A_{3},
$$

where

$$
A_{1}=a_{2}+a_{3}-a_{1}, A_{2}=a_{1}+a_{3}-a_{2}, A_{3}=a_{1}+a_{2}-a_{3} .
$$

In this case, the roots $\lambda_{i}$ must be chosen in order to verify the relation

$$
-8 \lambda_{1} \lambda_{2} \lambda_{3}=A_{1} A_{2} A_{3}
$$

We proceed by adapting the procedure used in case 2.i. According to (17), if $A_{1}, A_{2}$ and $A_{3}$ are non-zero then they must have the same sign, which means that one can define the real parameters

$$
\begin{aligned}
& j_{1}^{2}:=\frac{A_{1}}{A_{1}+A_{2}+A_{3}}=\frac{a_{2}+a_{3}-a_{1}}{a_{1}+a_{2}+a_{3}}, \\
& j_{2}^{2}:=\frac{A_{2}}{A_{1}+A_{2}+A_{3}}=\frac{a_{1}+a_{3}-a_{2}}{a_{1}+a_{2}+a_{3}}, \\
& j_{3}^{2}:=\frac{A_{3}}{A_{1}+A_{2}+A_{3}}=\frac{a_{1}+a_{2}-a_{3}}{a_{1}+a_{2}+a_{3}},
\end{aligned}
$$

verifying the relation $j_{1}^{2}+j_{2}^{2}+j_{3}^{2}=1$. Defining $a:=\left|a_{1}+a_{2}+a_{3}\right|$, (17) yields

$$
\lambda_{1}= \pm \frac{1}{2} a\left|j_{1} j_{2}\right|, \quad \lambda_{2}= \pm \frac{1}{2} a\left|j_{1} j_{3}\right|, \quad \lambda_{3}= \pm \frac{1}{2} a\left|j_{2} j_{3}\right| .
$$

In addition, we note that, according to relation (17), $\lambda_{1} \lambda_{2} \lambda_{3}$ has the opposite sign of $A_{1} A_{2} A_{3}$ and consequently the opposite sign of $a_{1}+a_{2}+a_{3}$. Thus, independently of the sign of $j_{1}, j_{2}$ and $j_{3}$ the following situations may be considered. If $a_{1}+a_{2}+a_{3}>0$ then $\lambda_{1} \lambda_{2} \lambda_{3}<0$, that is, $\lambda_{l}<0$ for all $l \in\{1,2,3\}$ or $\lambda_{l}<0$ for only one $l \in\{1,2,3\}$ and $\lambda_{k}>0$ for $k \neq l$. Analogously, if $a_{1}+a_{2}+a_{3}<0$ then $\lambda_{l}>0$ for all $l \in\{1,2,3\}$ or $\lambda_{l}>0$ for only one $l \in\{1,2,3\}$ and $\lambda_{k}<0$ for $k \neq l$. For simplicity, from now on we suppose that $j_{1}, j_{2}, j_{3}, a_{1}+a_{2}+a_{3}>0$ and that $\lambda_{1}, \lambda_{2}, \lambda_{3}<0$, i.e. we assume

$$
\lambda_{1}=-\frac{1}{2} a j_{1} j_{2}, \quad \lambda_{2}=-\frac{1}{2} a j_{1} j_{3}, \quad \lambda_{3}=-\frac{1}{2} a j_{2} j_{3} .
$$

By observing that

$$
\frac{a_{l}}{a_{1}+a_{2}+a_{3}}=\frac{1}{2}\left(1-j_{l}^{2}\right), \quad l \in\{1,2,3\},
$$


it is possible to express $P_{1}$ as

$$
\begin{aligned}
P_{1}\left(x_{0}, \underline{x}\right) & =x_{0}+\frac{1}{2}\left[\left(\left(1-j_{1}^{2}\right) x_{1}-j_{1} j_{2} x_{2}-j_{1} j_{3} x_{3}\right) e_{1}\right. \\
& +\left(-j_{1} j_{2} x_{1}+\left(1-j_{2}^{2}\right) x_{2}-j_{2} j_{3} x_{3}\right) e_{2} \\
& \left.+\left(-j_{1} j_{3} x_{1}-j_{2} j_{3} x_{2}+\left(1-j_{3}^{2}\right) x_{3}\right) e_{3}\right] .
\end{aligned}
$$

In other words

$$
P_{1}\left(x_{0}, \underline{x}\right)=x_{0}+d_{1} \underline{X}=x_{0}+\frac{1}{2}(\underline{x}-\underline{\tilde{X}}),
$$

where

$$
\underline{\tilde{X}}:=\left(j_{1} x_{1}+j_{2} x_{2}+j_{3} x_{3}\right)\left(j_{1} e_{1}+j_{2} e_{2}+j_{3} e_{3}\right)
$$

is such that

$$
\underline{X}=\frac{1}{2 d_{1}}(\underline{x}-\underline{\tilde{X}}) .
$$

Concerning the second degree polynomial $P_{2}\left(x_{0}, \underline{x}\right)$, we have proved that

$$
P_{2}\left(x_{0}, \underline{x}\right)=x_{0}^{2}+2 d_{1} x_{0} \underline{X}+d_{2} \underline{X}^{2}=x_{0}^{2}+x_{0}(\underline{x}-\underline{\tilde{X}})+\frac{1}{2}(\underline{x}-\underline{\tilde{X}})^{2} .
$$

Our aim now is to derive the general form of the coefficients $d_{s}$ in (12). Before we continue we need to prove an auxiliary result which will play an import role in the subsequent work.

Lemma 1 For a nonnegative integer $k$ the following relation holds

$$
\partial_{\underline{x}}(\underline{x}-\underline{\tilde{X}})^{k}= \begin{cases}-k(\underline{x}-\underline{\tilde{X}})^{k-1}, & k \text { even }, \\ -(k+1)(\underline{x}-\underline{\tilde{X}})^{k-1}, & k \text { odd }\end{cases}
$$

where $\underline{\tilde{X}}$ is the pseudo-complex first degree polynomial (21).

Proof:By recalling (19) and (20) we have that $\underline{x}-\underline{\tilde{X}}=w_{1} e_{1}+w_{2} e_{2}+w_{3} e_{3}$, where $w_{i}=w_{i}\left(x_{1}, x_{2}, x_{3}\right)$ are the real valued linear functions

$$
\begin{aligned}
& w_{1}=\left(1-j_{1}^{2}\right) x_{1}-j_{1} j_{2} x_{2}-j_{1} j_{3} x_{3} \\
& w_{2}=-j_{1} j_{2} x_{1}+\left(1-j_{2}^{2}\right) x_{2}-j_{2} j_{3} x_{3} \\
& w_{3}=-j_{1} j_{3} x_{1}-j_{2} j_{3} x_{2}+\left(1-j_{3}^{2}\right) x_{3} .
\end{aligned}
$$

If $k$ is even then both $(\underline{x}-\underline{\tilde{X}})^{k}$ and $(\underline{x}-\underline{\tilde{X}})^{k-2}$ are real valued homogeneous polynomials and therefore

$$
\begin{aligned}
\partial_{\underline{x}}(\underline{x}-\underline{\tilde{X}})^{k} & =\frac{k}{2}(\underline{x}-\underline{\tilde{X}})^{k-2} \partial_{\underline{x}}\left(-w_{1}^{2}-w_{2}^{2}-w_{3}^{2}\right) \\
& =k(\underline{x}-\underline{\tilde{X}})^{k-2}\left(-\left(w_{1} e_{1}+w_{2} e_{2}+w_{3} e_{3}\right)+\left(w_{1} j_{1}+w_{2} j_{2}+w_{3} j_{3}\right)\right) .
\end{aligned}
$$

Thus, after verifying that $w_{1} j_{1}+w_{2} j_{2}+w_{3} j_{3}=0$ the assertion follows immediately.

When $k$ is odd one has that $(\underline{x}-\underline{\tilde{X}})^{k-1}$ is real valued which leads to

$$
\begin{aligned}
\partial_{\underline{x}}(\underline{x}-\underline{\tilde{X}})^{k} & =\left[\partial_{\underline{x}}(\underline{x}-\underline{\tilde{X}})^{k-1}\right](\underline{x}-\underline{\tilde{X}})+(\underline{x}-\underline{\tilde{X}})^{k-1}\left[\partial_{\underline{x}}(\underline{x}-\underline{\tilde{X}})\right] \\
& =-(k-1)(\underline{x}-\underline{\tilde{X}})^{k-2}(\underline{x}-\underline{\tilde{X}})-2(\underline{x}-\underline{\tilde{X}})^{k-1} \\
& =-(k+1)(\underline{x}-\underline{\tilde{X}})^{k-1} .
\end{aligned}
$$


Proposition 4 A polynomial of the form

$$
P_{k}\left(x_{0}, \underline{x}\right)=\sum_{s=0}^{k}\left(\begin{array}{l}
k \\
s
\end{array}\right) a_{s} x_{0}^{k-s}(\underline{x}-\underline{\tilde{X}})^{s},
$$

where $a_{s} \in \mathbb{R}, a_{0}:=1$, and $\underline{\tilde{X}}:=\left(j_{1} x_{1}+j_{2} x_{2}+j_{3} x_{3}\right) \hat{\jmath}$, with $\hat{\jmath}^{2}=\left(j_{1} e_{1}+j_{2} e_{2}+j_{3} e_{3}\right)^{2}=-1$, is monogenic if and only if

$$
a_{s}=\frac{1}{2^{s}}\left(\begin{array}{c}
s \\
\left\lfloor\frac{s}{2}\right\rfloor
\end{array}\right)=c_{s}(2) .
$$

\section{Proof:}

We first prove that the form (24) of the coefficients $a_{s}$ is a necessary condition for $P_{k}$ being monogenic. According to (23), the action of the operator $\partial_{0}$ on $P_{k}$ gives

$$
\begin{aligned}
\partial_{0} P_{k}\left(x_{0}, \underline{x}\right)= & \sum_{s=0}^{k-1}(k-s)\left(\begin{array}{c}
k \\
s
\end{array}\right) a_{s}(k) x_{0}^{k-1-s}(\underline{x}-\underline{\tilde{X}})^{s} \\
= & \sum_{s=0}^{\left\lfloor\frac{k-1}{2}\right\rfloor}(k-2 s)\left(\begin{array}{c}
k \\
2 s
\end{array}\right) a_{2 s}(k) x_{0}^{k-1-2 s}(\underline{x}-\underline{\tilde{X}})^{2 s}+ \\
& +\sum_{s=0}^{\left\lfloor\frac{k}{2}\right\rfloor-1}(k-1-2 s)\left(\begin{array}{c}
k \\
2 s+1
\end{array}\right) a_{2 s+1}(k) x_{0}^{k-2-2 s}(\underline{x}-\underline{\tilde{X}})^{2 s+1} .
\end{aligned}
$$

Analogously, by means of Lemma 1 it follows that

$$
\begin{aligned}
\partial_{\underline{x}} P_{k}\left(x_{0}, \underline{x}\right)= & -\sum_{s=0}^{\left\lfloor\frac{k-1}{2}\right\rfloor}(2 s+2)\left(\begin{array}{c}
k \\
2 s+1
\end{array}\right) a_{2 s+1}(k) x_{0}^{k-1-2 s}(\underline{x}-\underline{\tilde{X}})^{2 s}+ \\
& -\sum_{s=0}^{\left\lfloor\frac{k}{2}\right\rfloor-1}(2 s+2)\left(\begin{array}{c}
k \\
2 s+2
\end{array}\right) a_{2 s+2}(k) x_{0}^{k-2-2 s}(\underline{x}-\underline{\tilde{X}})^{2 s+1} .
\end{aligned}
$$

Since $P_{k}$ is paravector-valued it is left and right monogenic, which means that, in particular, $\partial_{0} P_{k}\left(x_{0}, \underline{x}\right)=$ $-\partial \underline{x} P_{k}\left(x_{0}, \underline{x}\right)$. Thus, after comparing the real and vector parts of (25) and (26) one obtains, after simplification,

$$
a_{s}(k)=\left\{\begin{array}{lll}
\frac{2 s+1}{2 s+2} a_{s-1}(k), & s & \text { odd }, \\
a_{s-1}(k), & s & \text { even. }
\end{array}\right.
$$

By observing that $a_{0}(k)=1$, it follows immediately that $a_{s}(k)=c_{s}(2)$ (recall (3) for $n=2$ ).

We prove now that relation (24) implies the monogeinity of $P_{k}$. In this case, since

$$
\begin{aligned}
\partial_{0} P_{k}\left(x_{0}, \underline{x}\right) & =\sum_{s=0}^{k-1}(k-s) \frac{1}{2^{s}}\left(\begin{array}{c}
k \\
s
\end{array}\right)\left(\begin{array}{c}
s \\
\left\lfloor\frac{s}{2}\right\rfloor
\end{array}\right) x_{0}^{k-1-s}(\underline{x}-\underline{\tilde{X}})^{s} \\
& =k \sum_{s=0}^{k-1} \frac{1}{2^{s}}\left(\begin{array}{c}
k-1 \\
s
\end{array}\right)\left(\begin{array}{c}
s \\
\left\lfloor\frac{s}{2}\right\rfloor
\end{array}\right) x_{0}^{k-1-s}(\underline{x}-\underline{\tilde{X}})^{s}=k P_{k-1},
\end{aligned}
$$


it is sufficient to prove that $\partial_{\underline{x}} P_{k}=-k P_{k-1}$. From Lemma 1 one has that

$$
\begin{aligned}
\partial_{\underline{x}} P_{k}\left(x_{0}, \underline{x}\right)= & \sum_{s=1}^{\left\lfloor\frac{k}{2}\right\rfloor}\left(\begin{array}{c}
k \\
2 s
\end{array}\right)\left(\begin{array}{c}
2 s \\
s
\end{array}\right) \frac{-2 s}{2^{2 s}} x_{0}^{k-2 s}(\underline{x}-\underline{\tilde{X}})^{2 s-1}+ \\
& +\sum_{s=0}^{\left\lfloor\frac{k-1}{2}\right\rfloor}\left(\begin{array}{c}
k \\
2 s+1
\end{array}\right)\left(\begin{array}{c}
2 s+1 \\
s
\end{array}\right) \frac{-(2 s+2)}{2^{2 s+1}} x_{0}^{k-1-2 s}(\underline{x}-\underline{\tilde{X}})^{2 s} \\
= & \sum_{s=0}^{\left\lfloor\frac{k-2}{2}\right\rfloor}\left(\begin{array}{c}
k \\
2 s+2
\end{array}\right)\left(\begin{array}{c}
2 s+2 \\
s+1
\end{array}\right) \frac{-(s+1)}{2^{2 s+1}} x_{0}^{k-2-2 s}(\underline{x}-\underline{\tilde{X}})^{2 s+1}+ \\
& +\sum_{s=0}^{\left\lfloor\frac{k-1}{2}\right\rfloor}\left(\begin{array}{c}
k \\
2 s+1
\end{array}\right)\left(\begin{array}{c}
2 s+1 \\
s
\end{array}\right) \frac{-(s+1)}{2^{2 s}} x_{0}^{k-1-2 s}(\underline{x}-\underline{\tilde{X}})^{2 s} .
\end{aligned}
$$

Finally, it follows at once that $\partial_{\underline{x}} P_{k}=-k P_{k-1}$, since

$$
\begin{aligned}
(s+1)\left(\begin{array}{c}
k \\
2 s+2
\end{array}\right)\left(\begin{array}{c}
2 s+2 \\
s+1
\end{array}\right) & =k\left(\begin{array}{c}
k-1 \\
2 s+1
\end{array}\right)\left(\begin{array}{c}
2 s+1 \\
s
\end{array}\right) \\
(s+1)\left(\begin{array}{c}
k \\
2 s+1
\end{array}\right)\left(\begin{array}{c}
2 s+1 \\
s
\end{array}\right) & =k\left(\begin{array}{c}
k-1 \\
2 s
\end{array}\right)\left(\begin{array}{c}
2 s \\
s
\end{array}\right) .
\end{aligned}
$$

The characterization of all sequences of Appell polynomials of four variables is resumed in the following theorem.

Theorem 2 There exist exactly three different types of non-trivial and not degenerating to the 3D case Appell polynomials with four variables of the general form

$$
P_{k}\left(x_{0}, \underline{x}\right)=\sum_{s=0}^{k}\left(\begin{array}{l}
k \\
s
\end{array}\right) d_{s} x_{0}^{k-s}\left(X_{1} e_{1}+X_{2} e_{2}+X_{3} e_{3}\right)^{s},
$$

namely

1. Appell polynomials (SAP) of the form (2), i.e.

$$
P_{k}\left(x_{0}, \underline{x}\right)=\mathcal{P}_{k}^{n}\left(x_{0}, \underline{x}\right)=\sum_{s=0}^{k}\left(\begin{array}{l}
k \\
s
\end{array}\right) c_{s}(3) x_{0}^{k-s} \underline{x}^{s} .
$$

2. Appell polynomials (PCP) of the form (8), i.e.

$$
P_{k}\left(x_{0}, \underline{x}\right)=Z_{\hat{\imath}}^{k}=\sum_{s=0}^{k}\left(\begin{array}{l}
k \\
s
\end{array}\right) x_{0}^{k-s}\left(i_{1} x_{1}+i_{2} x_{2}+i_{3} x_{3}\right)^{s} \hat{\imath}^{s},
$$

where $\hat{\imath}^{2}=\left(i_{1} e_{1}+i_{2} e_{2}+i_{3} e_{3}\right)^{2}=-1$.

3. A third type of Appell polynomials of the form (23), i.e.

$$
P_{k}\left(x_{0}, \underline{x}\right)=\widetilde{P}_{k}\left(x_{0}, \underline{x}\right)=\sum_{s=0}^{k}\left(\begin{array}{l}
k \\
s
\end{array}\right) c_{s}(2) x_{0}^{k-s}(\underline{x}-\underline{\tilde{X}})^{s},
$$

where $\underline{\tilde{X}}:=\left(i_{1} x_{1}+i_{2} x_{2}+i_{3} x_{3}\right) \hat{\imath}$ and $\hat{\imath}^{2}=\left(i_{1} e_{1}+i_{2} e_{2}+i_{3} e_{3}\right)^{2}=-1$. 


\section{Acknowledgements}

This work was supported by Portuguese funds through the CIDMA - Center for Research and Development in Mathematics and Applications, the Research Centre of Mathematics of the University of Minho and the Portuguese Foundation for Science and Technology ("FCT - Fundação para a Ciência e a Tecnologia"), within projects PEst-OE/MAT/UI4106/2014 and PEstOE/MAT/UI0013/2014.

\section{References}

[1] Abul-Ez, M., Constales, D.: Basic sets of polynomials in Clifford analysis. Complex Variables, Theory Appl., 14, 177-185 (1990)

[2] Álvarez-Peña, C., Porter, M.: Appell bases for monogenic functions of three variables. Adv. in Appl. Clifford Algebras 23, 547-560 (2013)

[3] Appell, P.: Sur une classe de polynômes. Ann. Sci. École Norm. Sup. 9, no. 2, 119-144 (1880)

[4] Bock, S., Gürlebeck, K.: On a generalized Appell system and monogenic power series. Math. Methods Appl. Sci. 33, no. 4, 394-411 (2010)

[5] Brackx, F., Delanghe, R., Sommen, F.: Clifford analysis. Pitman, Boston-London-Melbourne, (1982)

[6] Cação, I., Falcão, M. I., Malonek, H. R.: Laguerre derivative and monogenic Laguerre polynomials: An operational approach. Math. Comput. Model. 53, no. 5-6, 1084-1094 (2011)

[7] Cação, I., Falcão, M. I., Malonek, H. R,.: Matrix representations of a special polynomial sequence in arbitrary dimension. Comput. Methods Funct. Theory, 12, 371-391 (2012)

[8] Cação, I., Malonek, H. R.: On complete sets of hypercomplex Appell polynomials. AIP Conference Proceedings (eds. Simos, Th. et al.) vol. 1048, 647-650 (2008)

[9] Carlson, B. C.: Polynomials satisfying a binomial theorem. J. Math. Anal. Appl. 32, 543-558 (1970)

[10] Cruz, C., Falcão, M.I., Malonek, H.R.: On the structure of generalized Appell sequences of paravector valued homogeneous monogenic polynomials. AIP Conference Proceedings (eds. Simos, Th. et al.) vol.1479 (1), 283-286 (2012)

[11] Cruz, C., Falcão, M.I., Malonek, H.R.: On pseudo-complex bases for monogenic polynomials. AIP Conference Proceedings (ed. S. Sivasundaram) vol. 1493 , 350-355 (2012)

[12] Cruz, C., Falcão, M.I., Malonek, H.R.: A note on totally regular variables and Appell sequences in hypercomplex function theory. Lecture Notes in Computer Science (eds. Murgante, B. et al.) 7971 (PART 1), 293-303 (2013)

[13] Cruz, C., Falcão, M.I., Malonek, H.R.: Monogenic pseudo-complex power functions and their applications. Mathematical Methods in the Applied Sciences, doi:10.1002/mma.2931 (2013)

[14] Delanghe, R.: On regular-analytic functions with values in a Clifford algebra. Math. Ann., 185, 91-111 (1970)

[15] Delanghe, R., Lávička, R., Souček, V.: The Gelfand-Tsetlin bases for Hodge-de Rham systems in Euclidean spaces. Math. Methods Appl. Sci. 7(35), 745-757 (2012)

[16] Delanghe, R., Sommen, F., Soucek, V.: Clifford algebra and spinor-valued functions. A function theory for the Dirac operator. Kluwer Academic Publishers (1992)

[17] Falcão, Cruz, J., M. I., Malonek, H. R.: Remarks on the generation of monogenic functions. 17th Inter. Conf. on the Appl. of Computer Science and Mathematics on Architecture and Civil Engineering, Weimar, (2006) 
[18] Falcão, M. I., Malonek, H. R.: Generalized exponentials through Appell sets in $\mathbb{R}^{n+1}$ and Bessel functions. AIP Conference Proceedings (eds. Simos, Th. et al.) vol. 936, 738-741 (2007)

[19] Fueter, R.: Über Funktionen einer Quaternionenvariablen. Atti Congresso Bologna 2, 145, (1930)

[20] Fueter, R.: Analytische Funktionen einer Quaternionenvariablen. Comment. Math. Helv. 4, 9-20 (1932)

[21] Fueter, R.: Functions of a Hyper Complex Variable, Lecture Notes written and supplemented by E. Bareiss, Fall Semester 1948/49. Univ. Zürich (1950)

[22] Gürlebeck, K., Malonek, H. R.: A hypercomplex derivative of monogenic functions in $\mathbb{R}^{n+1}$ and its applications. Complex Variables Theory Appl. 39, 199-228 (1999)

[23] Gürlebeck, K., Sprößig, W.: Quaternionic and Clifford Calculus for Physicists and Engineers. John Wiley \& Sons (1997)

[24] Gürlebeck, K., Habetha, K., Sprößig, W.: Holomorphic functions in the plane and $n$-dimensional space. Birkhäuser Verlag, Basel, (2008)

[25] Lávička, R.: Canonical bases for sl(2,c)-modules of spherical monogenics in dimension 3. Archivum Mathematicum 46 339-349 (2010)

[26] Malonek, H. R.: Rudolf Fueter and his motivation for hypercomplex function theory. Advances in Applied Clifford Algebras, 11, 219-229 (2001)

[27] Malonek, H. R.: A new hypercomplex structure of the euclidean space $\mathbb{R}^{m+1}$ and the concept of hypercomplex differentiabilit. Complex Variables 14 25-33 (1990)

[28] Malonek, H. R.: Selected topics in hypercomplex function theory. Clifford algebras and potential theory (S.-L. Eriksson, ed.), 7, University of Joensuu, 111-150 (2004)

[29] Malonek, H. R., De Almeida, R.: A note on a generalized Joukowski transformation. Appl. Math. Lett. 23 no.10, 1174-1178 (2010)

[30] Malonek, H. R., Falcão, M. I.: Special monogenic polynomials-properties and applications. AIP Conference Proceedings (eds. Simos, Th. et al.) vol. 936, 764-767 (2007)

[31] Malonek, H. R., Falcão, M. I.: 3D-mappings by means of monogenic functions and their approximation. Math. Methods Appl. Sci., 33, 423-430 (2010)

[32] Malonek, H.R., Falcão, M.I.: On paravector valued homogeneous monogenic polynomials with binomial expansion. Advances in Applied Clifford Algebras 22 (3, SI), 789-801 (2012)

[33] Stein, E. M., Weiss, G.: Generalization of the Cauchy-Riemann equations and representations of the rotation group. Amer. J. Math. 90, 163-196 (1968) 